\title{
CARACTERIZAÇÃO DO PERFIL COMUNICATIVO DE CRIANÇAS COM DISTÚRBIOS DO ESPECTRO AUTÍSTICO COM DIFERENTES INTERLOCUTORES
}

\author{
Characterizing the communicative profile of children \\ with autistic spectrum disturbances with different interlocutors
}

Aline Citino Armonia (1), Andréa Regina Nunes Misquiatti (2)

\begin{abstract}
RESUMO
Objetivo: caracterizar e comparar o perfil comunicativo de crianças com distúrbios do espectro autístico com dois diferentes interlocutores. Método: participaram da pesquisa nove sujeitos, sendo quatro crianças do gênero masculino, com faixa etária variando entre cinco anos e oito meses e onze anos, com distúrbios do espectro autístico e cinco terapeutas de linguagem. Foram realizadas análises do perfil comunicativo dessas crianças com os dois diferentes interlocutores (terapeuta de linguagem da própria criança e terapeuta desconhecida). Para análise dos dados foi empregado o Protocolo de Pragmática e tratamento estatístico dos resultados ( $p \leq 0,05$; Teste dos Postos Sinalizados de Wilcoxon). Resultados: foram levantados o número de atos comunicativos, o meio pelo qual esses atos foram expressos (vocal, verbal e gestual) e as funções comunicativas. Em média, o número de atos comunicativos expressos pelos sujeitos tanto com os terapeutas das próprias crianças $(5,10$ atos/min) como com a terapeuta desconhecida (4,93 atos/min) não foi estatisticamente significante. Observou-se na situação de interação com o próprio terapeuta que a média do percentual da ocorrência do meio comunicativo verbal $(31,50 \%)$ e a média de funções mais interativas $(33,25 \%)$ são maiores do que com terapeuta desconhecida. Conclusão: os diferentes interlocutores, estudados nesta pesquisa, não tiveram uma influência no perfil comunicativo destas crianças. Deste modo, sugeremse novos estudos, com inclusão de outros interlocutores, a fim de estabelecer quais variáveis interacionais interferem estatisticamente no perfil comunicativo de crianças do espectro autístico, com o intuito de contribuir para a elaboração de melhores propostas terapêuticas para esta população.
\end{abstract}

DESCRITORES: Transtorno Autístico; Relações Interpessoais; Linguagem Infantil; Estudos de Intervenção

\section{INTRODUÇÃO}

Os quadros do espectro autístico caracterizam-se por uma tríade de impedimentos graves e crônicos nas áreas de interação social, comunicação verbal e não verbal e interesses ${ }^{1}$. De acordo com a literatura, a linguagem é uma questão

(1) Fonoaudióloga da Associação de Pais e Amigos dos Excepcionais; Limeira, São Paulo, Brasil.

(2) Fonoaudióloga; Docente do Departamento de Fonoaudiologia da Universidade Estadual Paulista Júlio de Mesquita Filho, Marília, São Paulo, Brasil; Doutora em Lingüística pela Faculdade de Filosofia, Letras e Ciências Humanas da Universidade de São Paulo.

Conflito de interesses: inexistente central ${ }^{2-3}$ para a compreensão e intervenção das alterações de linguagem nesses quadros, principalmente no que se refere ao uso funcional da linguagem ${ }^{4-8}$, que está relacionado às habilidades de interação social ${ }^{2-9}$ e alterações no comportamento ${ }^{10}$.

Esses prejuízos representam um desvio em relação ao nível de desenvolvimento do indivíduo, afetando assim sua adaptação social, educacional e de comunicação ${ }^{11}$.

Podemos pensar na interação social como sendo uma ação entre sujeitos, e para que ela ocorra é essencial a existência da comunicação de uma linguagem, ou seja, de uma forma de construção e representação de mensagens ${ }^{12}$. 
Deste modo, o ambiente, o interlocutor e os comportamentos comunicativos não verbais, são variáveis importantes no estabelecimento da interação comunicativa ${ }^{13}$. Um estudo concluiu que 0 interlocutor adulto durante interações com crianças autistas provocou manifestações de comportamentos comunicativos e apenas a sua presença atraiu a atenção da criança, promovendo a iniciativa de comunicação ${ }^{13}$.

Neste contexto, o terapeuta da linguagem pode ser visto como um interlocutor ideal e mediador do processo terapêutico, uma vez que se molda ao paciente durante a interação, tornando-se um interlocutor mais simétrico. Assim, observou-se que terapeutas que atuam com crianças autistas não apresentam uma homogeneidade na atuação, 0 que evidencia uma diversidade interativa ${ }^{14}$.

Em outro estudo sob uma perspectiva pragmática, a interferência de diferentes interlocutores na comunicação da criança autista foi analisada ${ }^{15}$. O autor ${ }^{15}$, ao observar uma criança de oito anos de idade portadora de autismo, concluiu que seu comportamento pragmático variou em diversas situações comunicativas e com parceiros diferentes. Os resultados mostraram um maior número de funções comunicativas quando a interação era com um adulto familiar em relação a um adulto não familiar.

Diante do exposto, esta pesquisa tem como objetivo caracterizar o perfil comunicativo de crianças do espectro autístico com dois diferentes interlocutores propostos: terapeuta de linguagem do paciente e terapeuta desconhecida, a fim de comparar o desempenho comunicativo das diferentes díades.

\section{MÉTODO}

Trata-se de um estudo transversal, realizado em uma clínica - escola na cidade de Marília no estado de São Paulo. Participaram desta pesquisa quatro crianças com distúrbio do espectro autístico, todas do gênero masculino com faixa etária variando entre cinco anos e oito meses e seis anos e onze meses de idade (Figura 1), diagnosticadas por psiquiatra segundo os critérios específicos ${ }^{16,17}$.Como critério de inclusão nesta pesquisa, as crianças deveriam freqüentar atendimento fonoaudiológico, de terapia de linguagem, na clínica-escola com a mesma terapeuta há no mínimo três meses.

Para cada uma das quatro crianças participantes desta pesquisa foi proposta a utilização de dois diferentes interlocutores: terapeuta de linguagem (estagiário de fonoaudiologia) da referida criança na clínica-escola e um interlocutor desconhecido da criança (terapeuta de linguagem/estagiária de fonoaudiologia), sendo este o mesmo para todas as crianças, desta forma tendo a participação de cinco terapeutas de linguagem, totalizando nove participantes. Este segundo interlocutor, desconhecido da criança, é uma das autoras deste estudo, que previamente estudou os casos por meio dos prontuários e de supervisões com a professora supervisora do estágio, no qual as crianças receberam atendimento, e também autora desta pesquisa.

Os responsáveis foram informados sobre a realização da pesquisa, consultados sobre sua concordância em participar dela e assinaram o termo de consentimento livre e esclarecido.

A caracterização do perfil comunicativo das crianças foi realizada por meio do Protocolo de Pragmática ${ }^{18}$, sendo possível descrever o perfil comunicativo das crianças e analisar os aspectos funcionais da linguagem. Nesta análise verificouse o número de atos comunicativos por minuto, as funções comunicativas e o meio pelo qual esses atos foram expressos (vocal, verbal e gestual). Este protocolo consta de vinte categorias de funções comunicativas que, para análise dos resultados, foram divididas em mais interativas e menos interativas ${ }^{19}$, sendo consideradas as mais interativas: pedido de objeto, pedido de ação, pedido de rotina social, pedido de consentimento, pedido de informação, reconhecimento do outro, exibição, comentário, nomeação, exclamativa, narrativa, expressão de protesto, protesto, jogo compartilhado; e as menos interativas: reativa, performativa, não-focalizada, jogo, exploratória e auto-regulatória.

Nas sessões de filmagens foram utilizados brinquedos pertencentes à clínica-escola e filmadora. As gravações das situações de interação das crianças com os diferentes interlocutores tiveram a duração de 30 minutos. Os terapeutas das crianças

\begin{tabular}{|c|c|c|c|}
\hline Sujeito & Sexo & Idade & Diagnóstico \\
\hline 1 & Masculino & 5 anos e 8 meses & Síndrome de Asperger \\
\hline 2 & Masculino & 6 anos e 11 meses & Autismo \\
\hline 3 & Masculino & 5 anos e 4 meses & Autismo \\
\hline 4 & Masculino & 6 anos & Síndrome de Asperger \\
\hline
\end{tabular}

Figura 1 - Caracterização das crianças com distúrbios do espectro autístico 
somente receberam a orientação de preparar um atendimento com atividades e brinquedos escoIhidos pela própria criança, assim a gravação com a terapeuta desconhecida foi estruturada da mesma maneira, com os mesmos brinquedos, dando a ambos as mesmas possibilidades de interação.

Para o preenchimento do protocolo de pragmática foram utilizados 15 minutos de gravação de interação mais simétrica de cada filmagem, de acordo como procedimento de aplicação do protocolo ${ }^{18}$. Ocorreu uma gravação para cada interlocutor, somando duas gravações com cada criança e oito sessões de gravação ao todo na pesquisa, dentro do período de 12 meses. As duas gravações de cada criança foram realizadas em um período de no máximo um mês

As sessões de filmagens ocorreram durante situação de brincadeira livre. Em todas as gravações foram utilizados brinquedos lúdicos que proporcionassem uma situação de interação da criança com os interlocutores. Esses brinquedos foram escolhidos de acordo com a faixa etária e o interesse de cada criança, sendo utilizado o mesmo brinquedo com os dois diferentes interlocutores, oferecendo assim a mesma possibilidade de interação lúdica para todos.

Esta pesquisa foi aprovada pelo Comitê de Ética em Pesquisa da Faculdade de Filosofia e Ciências da Universidade Estadual Paulista - CEP/FFC/ UNESP-Marília - SP sob o protocolo n`3243/2008.

Também foi realizada a análise estatística dos dados encontrados, por meio do Teste dos Postos Sinalizados de Wilcoxon, a fim tanto de realizar a comparação dos resultados referentes ao perfil comunicativo com ambos os interlocutores, como de verificar média, desvio-padrão e significância.

\section{RESULTADOS}

Conforme demonstrado na Tabela 1, verificouse, a partir do teste de Wilcoxon, que, em média, não houve diferença estatisticamente significante $(p \leq 0,05)$ no número de atos comunicativos expressos pelos sujeitos em ambas as situações (com a própria terapeuta e com a terapeuta desconhecida).

No entanto, observou-se que o sujeito 4 apresentou um número de atos comunicativos por minuto maior com o próprio terapeuta $(7,73)$ quando comparado com a terapeuta desconhecida $(4,8)$. Diferentemente, o sujeito 3 apresentou um maior número de atos comunicativos por minutos com a terapeuta desconhecida $(6,4)$ em relação ao próprio terapeuta $(3,66)$.

Tabela 1 - Médias do número de atos comunicativos por minuto de cada sujeito com os dois interlocutores

\begin{tabular}{ccc}
\hline Sujeitos & Terapeuta do paciente & $\begin{array}{c}\text { Terapeuta desconhecida } \\
\text { do paciente }\end{array}$ \\
\hline 1 & 5,8 & 4,13 \\
2 & 3,2 & 4,4 \\
3 & 3,66 & 6,4 \\
4 & 7,73 & 4,8 \\
Média & 5,10 & 4,93 \\
Desvio-padrão & 2,09 & 1,02 \\
Significância & 0,715 & 0,715 \\
\hline
\end{tabular}

Na Tabela 2, podemos observar que a média do percentual da ocorrência dos meios comunicativos de cada sujeito com os dois diferentes interlocutores não foi estatisticamente significante $(p \leq 0,05)$.

Nota-se que a média do percentual da ocorrência do meio comunicativo verbal em todos os sujeitos é maior na situação de interação com o próprio terapeuta $(31,50 \%)$. Já a média do percentual de ocorrência do meio gestual é semelhante nas duas situações de interação: com o próprio terapeuta $(90,25 \%)$ e com terapeuta desconhecida $(93,25 \%)$. O meio vocal apresentou uma média maior na situação de interação com o interlocutor desconhecido $(31,50 \%)$ em relação ao próprio terapeuta $(16 \%)$.

Conforme descrito na Tabela 3, a média do percentual das funções comunicativas mais e menos interativas expressas por cada sujeito com os dois diferentes interlocutores não foi estatisticamente significante $(p \leq 0,05)$. 
Tabela 2 - Percentual da ocorrência dos meios comunicativos de cada sujeito com os dois interlocutores

\begin{tabular}{ccccccc}
\hline & \multicolumn{7}{c}{ Meios comunicativos } \\
\cline { 2 - 7 } Sujeitos & \multicolumn{2}{c}{ Verbal (\%) } & \multicolumn{2}{c}{ Vocal (\%) } & \multicolumn{2}{c}{ Gestual (\%) } \\
\cline { 2 - 6 } & Terapeuta & $\begin{array}{c}\text { Terapeuta } \\
\text { desconhecida } \\
\text { do paciente }\end{array}$ & Terapeuta & $\begin{array}{c}\text { Terapeuta } \\
\text { desconhecida } \\
\text { do paciente }\end{array}$ & $\begin{array}{c}\text { Terapeuta } \\
\text { Terapeuta }\end{array}$ & $\begin{array}{c}\text { Tesconhecida } \\
\text { do paciente }\end{array}$ \\
\hline 1 & $21 \%$ & $13 \%$ & $40 \%$ & $40 \%$ & $91 \%$ & $85 \%$ \\
2 & $19 \%$ & $3 \%$ & $6 \%$ & $50 \%$ & $94 \%$ & $97 \%$ \\
3 & $36 \%$ & $33 \%$ & $16 \%$ & $36 \%$ & $87 \%$ & $97 \%$ \\
4 & $50 \%$ & $44 \%$ & $2 \%$ & 0 & $89 \%$ & $94 \%$ \\
Média & $31,50 \%$ & $23,25 \%$ & $16 \%$ & $31,50 \%$ & $90,25 \%$ & $93,25 \%$ \\
Desvio-padrão & $14,48 \%$ & $18,63 \%$ & $17,05 \%$ & $21,81 \%$ & $2,99 \%$ & $5,68 \%$ \\
Significância & 0,068 & 0,068 & 0,285 & 0,285 & 0,465 & 0,465 \\
\hline
\end{tabular}

Tabela 3 - Percentual das funções comunicativas mais e menos interativas expressas por cada sujeito com os dois interlocutores

\begin{tabular}{ccccc}
\hline & \multicolumn{4}{c}{ Funções comunicativas } \\
\cline { 2 - 5 } & Mais interativas & \multicolumn{2}{c}{ Menos interativas } \\
\cline { 2 - 5 } & Terapeuta & $\begin{array}{c}\text { Terapeuta } \\
\text { desconhecida } \\
\text { do paciente }\end{array}$ & Terapeuta & $\begin{array}{c}\text { Terapeuta } \\
\text { desconhecida } \\
\text { do paciente }\end{array}$ \\
\hline 1 & $9 \%$ & $11 \%$ & $91 \%$ & $89 \%$ \\
2 & $37 \%$ & $35 \%$ & $63 \%$ & $65 \%$ \\
3 & $33 \%$ & $19 \%$ & $67 \%$ & $81 \%$ \\
4 & $54 \%$ & $51 \%$ & $46 \%$ & $49 \%$ \\
Média & $33,25 \%$ & $29 \%$ & $66,75 \%$ & $71 \%$ \\
Desvio-padrão & $18,55 \%$ & $17,74 \%$ & $18,55 \%$ & $17,74 \%$ \\
Significância & 0,197 & 0,197 & 0,197 & 0,197 \\
\hline
\end{tabular}

Apesar dos resultados não apresentarem significância estatística, demonstram que a média de funções mais interativas expressas na situação de interação com o próprio terapeuta (33,25\%) foi maior do que a média na situação de interação com a terapeuta desconhecida (29\%). O contrário ocorreu com a média das funções menos interativas, que foi maior na situação com a terapeuta desconhecida $(71 \%)$ do que na situação com o interlocutor conhecido, o próprio terapeuta $(66,75 \%)$.

\section{DISCUSSÃO}

Os dados deste estudo indicam que o segundo interlocutor proposto na pesquisa, mesmo sendo desconhecido da criança, assume uma postura considerada ideal, utilizando de recursos teóricos para facilitar a interação, corroborando outros estudos ${ }^{14}$, em que o terapeuta foi visto como interlocutor ideal e também como elemento mediador do processo terapêutico.

Nesta pesquisa o principal foco de investigação foi o perfil comunicativo com os diferentes interlocutores, não sendo observada diferença estatisticamente significante nas diferentes situações de interação; contrariamente ao que foi apontado pela literatura, que sugere que o contexto interacional pode ter maior influência do que o contexto ambiental ${ }^{6}$ e que um dos fatores que interferem no perfil comunicativo de crianças do espectro autístico é a interferência de diferentes interlocutores ${ }^{20}$.

Em conformidade com outros estudos ${ }^{21,22}$, o percentual de ocorrência do meio comunicativo gestual foi maior do que o verbal e vocal. Pode-se observar ainda que a média do meio comunicativo verbal foi maior com o próprio terapeuta, enquanto que o meio vocal foi maior com a terapeuta desconhecida. A maior ocorrência do meio vocal deu-se 
possivelmente devido ao maior uso de funções comunicativas menos interativas (como reativa e não focalizada), que foram expressas pelos meios vocal e gestual.

Outros autores ${ }^{23}$ observaram maior progresso no uso do meio vocal e gestual de crianças autistas em interação com suas mães durante um período de seis meses, sugerindo que a interação com interlocutor familiar proporciona maior segurança para a sua utilização nos momentos em que são necessários.

A média de funções comunicativas mais interativas foi maior na situação de interação com o próprio terapeuta da criança, mostrando que o perfil comunicativo nesta situação foi melhor em termos de qualidade, sendo observada uma maior interação e um melhor desempenho destas crianças quando próximas a um interlocutor familiar, apoiando a literatura que revela que a intervenção terapêutica de linguagem necessita levar em conta o contexto comunicativo em que a comunicação se estabelece ${ }^{19-20}$.

É possível notar ainda que, embora a diferença do perfil comunicativo de crianças do espectro autístico com diferentes interlocutores não seja estatisticamente significante, observa-se uma diferença na qualidade destas situações de interação, sugerindo assim que a presença de um interlocutor conhecido favorece o aparecimento de funções mais interativas. Assim, este dado indica que o tempo de intervenção fonoaudiológica com o mesmo terapeuta pode favorecer a interação entre ambos e predizer um melhor resultado deste processo, como destacado por outros autores ${ }^{23}$.
Observa-se também que as médias das funções comunicativas expressas apresentaram maior ocorrência de funções menos interativas, independentemente do interlocutor, comprovando os achados da literatura ${ }^{24}$.

\section{CONCLUSÃO}

Esta pesquisa permitiu caracterizar o perfil comunicativo de quatro crianças com distúrbio do espectro autístico com dois diferentes interlocutores propostos: terapeuta de linguagem da própria criança e terapeuta de linguagem desconhecida da criança, no entanto, não foram observadas diferenças estatisticamente significantes no perfil comunicativo entre as díades.

Vale ressaltar que devido ao pequeno número de sujeitos estudados, os resultados aqui encontrados são limitados e não devem ser generalizados. Assim, sugere-se que sejam realizados novos estudos com uma maior população e com a inclusão de outros interlocutores, a fim de contribuir para a elaboração de melhores propostas terapêuticas para esta população.

\section{AGRADECIMENTOS}

À fonoaudióloga Maria Cláudia Brito, doutoranda em Educação pela Universidade Estadual Paulista, UNESP, Marília pela contribuição neste estudo.

Às fonoaudiólogas Carolina Roganti Leite Moreira e Ana Paula Marcelino. 


\section{ABSTRACT}

Purpose: characterize and compare the communicative profile of children with disturbances of autistic specter with two different speakers. Method: nine subjects took part in the research, among them four children of the masculine gender, between five years old and eight months and eleven years old, with disturbances of the autistic specter and five speech and language therapists. We carried out an analysis of the communicative profile respective to these children with two different speakers (proper child's speech and language and unknown therapist). For the analysis of the information we used the Protocol of Pragmatica and statistical treatment of the results ( $p \leq 0.05$; Tests of Wilcoxon's Signalized Posts). Results: we raised the number of the communicative acts, the mean through those acts was expressed (vocal, verbal and signal) and also the communicative functions. As a rule, the number of the communicative acts expressed by subjects as with proper child's therapists $(5,10$ acts/ $\mathrm{min}$ ) as with unknown therapist $(4,93 \mathrm{acts} / \mathrm{min})$ was not statistically significant. It was observed in the situation of interaction with proper therapist that the mean value for the percentage of occurrence of verbal communicative mean (31.50\%) and the average of more interactive functions $(33.25 \%)$ are larger than unknown therapist. Conclusion: the different speakers, studied in this research, do not have influence on the communicative profile of these children. From that, we suggest new studies, with inclusion of other speakers, in order to settle which interactive variables interfere statistically in the children's communicative profile of the autistic specter intention to contribute to the elaboration of better therapeutic offers for these people.

KEYWORDS: Autistic Disorder; Interpersonal Relations; Child Language; Intervention Studies

\section{REFERÊNCIAS}

1. Tamanaha AC, Perissinoto J, Chiari BM. Uma breve revisão história sobre a construção dos conceitos do Autismo Infantil e da síndrome de Asperger. Rev Bras Fonoaudiol. 2008; 13 (3): 296-9.

2. Jones LA, Campbell JM. Clinical Characteristics Associated with language regression for children with autism spectrum disorders. J Autism Dev Disord. 2010 Jan; 40(1): 52-62.

3. Klin A, Saulnier C, Sparrow S, Cicchetti D, Volkmar F, Lord C. Social and communication abilities and disabilities in higher functioning individuals with autism spectrum disorders: The vineland and the ADOS. Journal of Autism and Developmental Disorders. 2007; 37:748-59.

4. Seung HK, Ashwell S, Elder JH, Valcante G. Verbal communication outcomes in children with autism after in-home father training. Journal of Intellectual Disability Research. 2006; 5(2):139-50.

5. Volden J, Coolican J, Garon N, White J, Bryson $\mathrm{S}$. Brief report: pragmatic language in autism spectrum disorder: relationships to measures of ability and disability. J Autism Dev Disord. 2009 Feb;39(2):388-93.

6. Misquiatti ARN. A interferência do contexto ambiental no desempenho funcional na comunicação de crianças Transtornos do Espectro
Autístico [dissertação]. São Paulo: Faculdade de Medicina da Universidade de São Paulo; 2006.

7. Beldaki A. Language impairments in autism: evidence against mind-blindness. SOAS Working Papers in Linguistic. 2006; 14:3-13.

8. Perissinoto J. Linguagem da criança com autismo. In: Perissinoto J. (Org). Conhecimentos essenciais para atender bem as crianças com autismo. São José dos Campos: Pulso, 2003, cap.4, p. 39-44.

9. Drew A, Baird G, Taylor E, Milne E, Charman T. The social communication assessment for toddlers with autism (SCATA): an instrument to measure the frequency, form and function of communication in toddlers with autism spectrum disorder. J Autism Dev Disord. 2007; 37:648-66.

10. Ketelaars MP, Cuperus J, Jansonius K, Verhoeven L. Pragmatic language impairment and associated behavioural problems. Int $\mathrm{J}$ Lang Commun Disord. 2010 Mar; 45(2):204-14.

11. Silva RA, Lopes-Herrera AS, De Vitto LPM. Distúrbio de linguagem como parte de um transtorno global do desenvolvimento: descrição de um processo terapêutico fonoaudiológico. Rev Bras Fonoaudiol. 2007; 12 (4): 322-8.

12. Passerino LM, Santarosa LCM. Interação social no autismo em ambientes digitais de aprendizagem. Psicol Reflex Crit. 2007; 20 (1): 54-64.

13. Farah LSD, Perissinoto, J, Chiari, BM. Estudo longitudinal da atenção compartilhada em crianças 
autistas não verbais. Revista CEFAC 2009; 11(4): 587-97.

14. Miilher LP, Fernandes FDM. Análise das funções comunicativas expressas por terapeutas e pacientes do espectro autístico. Pró-Fono Rev Atual Cient. 2006; 18 (3): 239-48.

15. Bernard-Optiz V. Pragmatic analysis of thee communicative behavior of an autistic children. $J$ Speech Hear Dis. 1982; 47 (1): 99-109.

16. American Psychiatry Association. Manual diagnóstico estatístico de transtornos mentais. (DSM-IV-TR). Porto Alegre: Artes Médias; 2002

17. OMS (Organização mundial da saúde). Classificação de transtornos mentais e de comportamento da CID-10: Descrições clínicas e diretrizes diagnósticas. Porto Alegre:Artes Médicas; 1993.

18. Fernandes FDM. Pragmática. In: Andrade CRF, Befi-Lopes DM.; Fernandes FDM, Wertznner, HF. ABFW: Teste de linguagem infantil nas áreas de fonologia, vocabulário, fluência e pragmática. Barueri: Pró-Fono, 2004.

19. Fernandes FDM. Atuação fonoaudiológica com crianças com transtornos do espectro autístico [tese de livre docência]. São Paulo: Faculdade de Medicina da Universidade de São Paulo; 2002.
20. Cardoso C, Fernandes FDM. A comunicação de crianças do espectro autístico em atividades em grupo. Pró-Fono Rev Atual Cient. 2004; 16 (1): 67-74.

21. Campelo LD, Lucena JÁ, Lima CN, Araújo HMM, Viana LGO, Veloso MML, Correia PIFB, Muniz LF. Autismo: um estudo de habilidades comunicativas em criança. Rev CEFAC. 2009; 11(4): 598-606 .

22. Fernandes FDM, Molini-Avejonas DR, SousaMorato PF.Perfil funcional da comunicação nos distúrbios do espectro autístico. Rev CEFAC. 2006; $8(1): 20-6$

23. Fernandes FD, Cardoso C, Sassi FC, Amato $\mathrm{CH}$, Sousa-Morato PF. Fonoaudiologia e autismo: resultado de três diferentes modelos de terapia de linguagem. Pró-Fono Rev Atual Cient. 2008; 20(4):267-72.

24. Britto MC, Misquiatti ARN, Carrara K. Perfil comunicativo de crianças com transtorno do espectro autístico e em desenvolvimento normal durante interação com suas mães. In: Almeida MA, Mendes EG, Hayaashi MCPI. Temas em Educação Especial: Múltiplos olhares. Araraquara, SP: Junqueria \& Main: Brasília. DF: CAPES, 2008; 381-90.
http://dx.doi.org/10.1590/S1516-18462011005000043

RECEBIDO EM: 07/05/2010

ACEITO EM: 02/02/2011

Endereço para correspondência:

Andréa Regina Nunes Misquiatti

Av. Hygino Muzzi Filho, 737 - Caixa Postal 181

Marília - SP - Brasil

CEP: 17525-050

E-mail: amisquiatti@uol.com.br 